\title{
Enhancement of Function of the Prefrontal Cortex to Improve Symptoms of Schizophrenia
}

\section{Prajjita Sarma Bardoloi*}

Psychiatrist, Queen Elizabeth Hospital II, Grande Prairie, Alberta, Canada

*Corresponding Author: Prajjita Sarma Bardoloi, Psychiatrist, Queen Elizabeth

Hospital II, Grande Prairie, Alberta, Canada.

DOI: $10.31080 /$ ASNE.2020.03.0183
Received: April 01, 2020

Published: April 28, 2020

(C) All rights are reserved by Prajjita Sarma

Bardoloi.

\section{Abstract}

Schizophrenia is a chronic psychotic illness with multifactorial etiology that manifests with a constellation of positive, negative, affective and cognitive symptoms [1]. As we are learning more about the neurobiology of schizophrenia, the role of the prefrontal cortex (PFC) in schizophrenia has become more evident. While various medications are available to treat psychotic symptoms in schizophrenia, treatment of cognitive functions, residual symptoms and comorbid disorders is still a substantial challenge. Here we present a case of a young male who suffers from schizophrenia, with a previous diagnosis of ADHD and substance abuse, who showed remarkable improvement following treatment with the alpha-2A adrenergic agonist guanfacine, likely related to enhanced PFC function.

Keywords: Schizophrenia; Prefrontal Cortex (PFC); Dopaminergic (DA)

\section{Background}

Neurobiology of schizophrenia

Schizophrenia is a brain disorder with multifactorial etiology, manifesting in young adulthood when exposed to triggers which unveil the disease process. The illness likely starts in utero, but the symptoms manifest in late adolescence or young adulthood, likely due to environmental triggers. Pre- and post-natal exposure to toxins or infectious agents, autoantibodies, traumatic events or stress can negatively influence the grey and white matter maturational process of the brain, including apoptosis, myelination and synaptogenesis [2,3]. This may result in defective cortical neuronal migration [4] leading to error of connectivity between various brain regions, including midbrain, nucleus accumbens, thalamus, temporo-limbic and prefrontal cortex [5-7]. The inadequately connected brain regions and the networks are vulnerable to stress, and the developmental processes like synaptic pruning, myelination and endocrinal changes during puberty [8]. Along with the defective networks, there is also excessive dopaminergic (DA) activity in the caudate which may aggravate positive symptoms (delusions and hallucinations). Disturbance in the dorsolateral PFC in schizophrenia causes working memory deficits $[9,10]$. Cognitive symptoms in schizophrenia including thought disorder have been linked to poor PFC functioning (executive dysfunction), including strong correlations between thought disorders, working memory deficits and hypofrontality $[2,9,11]$.
According to the "dual hit" hypothesis, people with schizophrenia are exposed to two important periods relevant to the etiology of their illness. First one is during perinatal period which includes genetic and early environmental factors (e.g. maternal infection, maternal malnutrition, immune dysfunction, hypoxia) that renders their brain vulnerable to develop schizophrenia later in life $[9,12,13]$. Symptoms of schizophrenia unmask during young adulthood, when they are hit by the second wave of stress that includes intrinsic factors like puberty and hormonal changes and external factors like stress, substance abuse etc [9]. Post-mortem studies of schizophrenia have found that layer III pyramidal cells are especially vulnerable in this disorder [8]. As described below, the unique molecular structure of the layer III pyramidal cells of the PFC renders themselves vulnerable and may get easily disrupted during stress.

\section{Role of prefrontal cortex}

The PFC is like the air traffic controller in a busy airport: it controls the highly coordinated functions of the brain, bolstering relevant signals and inhibiting unwanted information and reactions through complex and extensive network connections. In this way, PFC networks generate the executive functions e.g. working memory, impulse control, attention regulation, mental flexibility, planning and organization, as well as metacognitive abilities such as insight and judgment $[14,15]$. The PFC provides top down control 
for smooth hierarchical functioning of the brain [16,17]. However, the PFC is very sensitive to the neurochemical environment, and even modest changes in catecholamine levels can cause significant changes in its functioning [14,16]. The catecholamines Dopamine (DA) and norepinephrine (NE) have a critical, "inverted U" influence on PFC functioning [18]. DA is an essential neuromodulator in the PFC through its actions at D1 receptors. Either too much or too little stimulation of D1 receptors can impair PFC functioning [18]. Thus, low dose D1R agonists can improve working memory and attention regulation, but excessive DA released during stress exposure can impair PFC functioning. Similarly, low to moderate levels of NE augments PFC function, while high level of NE released during stress arrests $\mathrm{PFC}$ function. Moderate amounts of NE engage high affinity, $\boldsymbol{\alpha} 2 \mathrm{~A}$ adrenoreceptors and improve PFC functions. In contrast, very high levels of NE release during stress engage low affinity, $\boldsymbol{\alpha} 1$ adrenoreceptors, impairing PFC functions [9,18-23].

These catecholamine actions are now understood at the cellular level. The working memory functions arise from pyramidal cells in layer III of the dIPFC (dorsolateral prefrontal cortex) that can generate and maintain mental representations with their rich horizontal connections. These neurons are also known as delay cells because of this unique property where they can represent information over a delay in a working memory task. These recurrent excitatory circuits rely on NMDAR to maintain information in working memory. Thus, these cells can act like a memory pad that can maintain information without any external stimuli [24]. The tuning of these recurrent excitatory networks is refined by parvalbumin containing GABA interneurons via lateral inhibition $[23,25]$.

Dynamic network connectivity: The catecholamines have an important role in modulating the connectivity of the layer III pyramidal cell networks that generate mental representations. These circuits contain the molecular machinery to magnify calcium-cAMP signaling near NMDAR synapses on spines, which opens nearby potassium $\left(\mathrm{K}^{+}\right)$channels to weaken connectivity and reduce firings [18]. These signaling mechanisms are called dynamic network connectivity (DNC) and play a vital role in the adequate functioning of the PFC $[9,26]$. Various post-mortem studies indicated an important role of abnormal cAMP-Ca signaling in the DLPFC in schizophrenia. In a normal physiological state, feed-forward calcium- cAMP-PKA signaling opens $\mathrm{K}^{+}$channels (HCN and KCNQ) as negative feedback in a recurrent excitatory circuit to protect against seizures. However, these signaling pathways are also driven by stress exposure, where they cause a loss of dIPFC neuronal firing and impaired cognitive function [16,27]. In schizophrenia the prefrontal networks get impaired by stress via D1 receptor activation by cAMP-PKA signaling [28]. There are several important factors that regulate calcium- cAMP-PKA signaling and help maintain normal physiology. For example, cAMP signaling can be inhibited by post-synaptic mGluR3 or $\alpha 2 \mathrm{~A}-\mathrm{AR}$ on the dendritic spine, while phosphodiesterase 4A (PDE4A) catabolizes cAMP and is anchored in the correct position by DISC $1[9,29,30]$. These regulatory events are critical for strong dIPFC connections.

Role of pharmacotherapy to improve PFC: Abnormalities in DNC regulation and overactive feedforward calcium- cAMP-PKA$\mathrm{K}^{+}$signaling can reduce $\mathrm{PFC}$ neuronal firing and lead to symptoms of poor PFC functioning or hypofrontality, which then manifests into various psychiatric disorders. A novel approach initiated by the Arnsten Lab at Yale University was to use the $\boldsymbol{\alpha} 2 \mathrm{~A}-\mathrm{AR}$ agonist, guanfacine, as a neuroprotective agent that inhibits calcium- cAMP$\mathrm{PKA}-\mathrm{K}^{+}$signaling, enhances task-related firing and improves cognitive performance. Guanfacine was also found to be protective towards dendritic spines and cognitive functions in the face of chronic stress, as well as have anti-inflammatory properties $[9,27,29,30]$. The $\boldsymbol{\alpha} 2 \mathrm{~A}$ adrenoreceptor agonist improves working memory and behavioral inhibition and prevents distractibility by improving topdown control by PFC. It improves attention and reduces aggressive behavior. It may reduce anxiety by enhancing PFC regulation of limbic structures such as the amygdala, as well as directly reducing the stress response and weakening amygdala actions. In human trials, guanfacine improves involuntary movements like motor and vocal tics and used to treat Tourette's syndrome [18]. It is approved by the FDA for treating ADHD in children. The current case history provides an example where guanfacine aided a young man with a history of ADHD and substance abuse and emergent psychotic features consistent with schizophrenia.

\section{Case Presentation}

Patient is a 26-year-old single Caucasian male, who lives with his parents. He is unemployed and is on social assistance.

Clinical history: He was diagnosed with schizophrenia at the age of 22. His first psychotic episode was at the age of 21, when he travelled to Asia with his girlfriend. During his trip, he started experiencing auditory hallucinations and paranoid persecutory delusions. Although he had a history of using drugs in the past, he denied using any drugs at that time and thought his symptoms were from the antimalarial medication he took prior to travelling. His symptoms of hallucination and paranoid delusions persisted for about 5 weeks until he came back to Canada and was started on Olanzapine. Although he improved with medications for some time, he continued to struggle and was eventually diagnosed with schizophrenia.

This patient also has a history of drug use and he tried several illicit drugs including MDMA, LSD, DMT, Cocaine, methamphetamine 
and marijuana in the past when he was a teenager. He continued using methamphetamine off and on, with regular use of marijuana.

Past psychiatric history was positive for ADHD, and he was prescribed Adderall XR 20 mg daily for about 4 years, which was stopped when he developed psychosis. After his diagnosis of schizophrenia, he was admitted to the hospital twice with psychosis and was tried on olanzapine and aripiprazole with good outcomes. However, as he was not compliant with his oral medications, he was put on a monthly injection of paliperidone. Despite being on antipsychotic medication, he continued to experience some residual symptoms in the form of auditory hallucinations and paranoid delusions. He also had significant anxiety and he complained of his mind being "noisy". He also continued to use marijuana that he attributed to use for his anxiety.

His previous diagnoses were- Schizophrenia, ADHD and polysubstance use disorder, including use of MDMA, LSD, DMT, cocaine, methamphetamine and marijuana. He also continued to struggle with symptoms of impaired attention that interfered with his work. He enjoyed working as a cook but could not continue as he was easily distracted in a loud environment.

Family History was positive for Bipolar disorder and alcoholism in his father.

Patient was born 1 week post-dated, born after being induced. He does not recall delayed speech or development. There was no history of emotional or physical abuse. He struggled through school due to his poor attention span and distractibility. He did not like his core subjects but enjoyed music. He was able to complete high school and then worked a few jobs and enjoyed working as a cook, but he was unable to hold a job for sustained periods due to his poor attention. He had a girlfriend for about 4 years, but the relationship ended after he became psychotic.

Key points:

- Born with some genetic vulnerability for developing a psychiatric disorder from a positive family history.

- History of ADHD, a neurodevelopmental disorder with poor functioning of the dorsolateral PFC \& poor executive function (poor attention, poor decision making, poor impulse control) and poor insight, which likely contributed to:

- Use of illicit drugs at an early age causing additional destruction of neurons.

- Continued use of marijuana, likely contributing to residual symptoms.

- Non-compliance with medication.

- Combinations of the above factors have likely helped unmasking the symptoms of schizophrenia as well as in perpetuating the disease.

\section{Investigations}

Patient's physical examination, laboratory investigations and imaging studies were unremarkable.

\section{Treatment}

Goals of treatment

Treatment of core symptoms and improvement of residual symptoms by:

- $\quad$ Continuing treatment of psychotic symptoms by using long acting depot antipsychotic injection.

- Using medications to improve PFC function that in turn will improve executive functions and insight. This will likely help with abstinence from illicit drugs improve functionality and compliance with medication.

Patient agreed to start on guanfacine (Intuniv XR) to improve his PFC functioning. He was started on $1 \mathrm{mg}$ at bedtime and the dose was gradually increased to $3 \mathrm{mg}$. As he weighs 68 kilograms, this was about $0.05 \mathrm{mg} / \mathrm{kg}$ dosage. This was added in conjunction with an injection of Paliperidone $100 \mathrm{mg}$ i/m every month.

\section{Outcome and follow-up}

Patient reported noticing a significant improvement in his symptoms on review at 3 months after starting guanfacine treatment. He reported feeling "very calm" and did not have the background distracting noises that had been so problematic for so many years. He was able to stay abstinent from using any street drugs. His residual psychotic symptoms also resolved. His ADHD symptoms improved as well. He was compliant with his medications as he had the insight to know that they were very helpful.

Brief psychiatric rating scale scores:

- $\quad$ Prior to starting Guanfacine XR was - 42

- 3 months after starting Guanfacine XR- 19.

His overall functioning also improved. He started attending vocational programs to prepare for employment.

\section{Discussion}

This case is a typical example of schizophrenia, with a previous diagnosis of ADHD and substance abuse complicating his management. There are several aspects to consider in his case, which are: His initial diagnosis of ADHD, a neurodevelopmental disorder which involves impaired PFC functioning and top down control. ADHD like symptoms may often present during the prodromal period of schizophrenia and may actually be a prodrome rather than a separate diagnosis. Poor PFC functioning also presents 
with impaired executive function (e.g. impaired working memory, response inhibition and insight). These factors made him more vulnerable to substance-abuse. With predisposing genetic factors and a vulnerable brain, this patient's substance abuse (mostly stimulant and Marijuana) most likely unmasked his schizophrenia symptoms. Although his positive symptoms of schizophrenia improved partially with antipsychotic medications, he continued to have some residual symptoms. The addition of guanfacine treatment strengthened his PFC function which resulted in better executive function. As a result, he had improved attentional control and insight, and did not feel the need to use any illicit drugs, including marijuana to self-medicate.

\section{Learning Points/Take Home Messages}

- Although we have come a long way in treating schizophrenia but are still in great need of treatments to improve life of a schizophrenic individual.

- Restoration of cognition and executive function can allow a patient to have better control of their own lives.

- This case illustrates a novel approach to treatment based on the neurobiology, where strengthening the PFC can improve treatment outcome for patients with schizophrenia.

\section{Conclusion}

Schizophrenia is a chronic psychotic illness that manifests with a constellation of positive, negative, affective and cognitive symptoms. Brain's prefrontal cortex plays an important role in the cognitive symptoms of this illness. Treatment of cognitive symptoms shows improvement of overall outcome and should be included as an important step in management of schizophrenia patients.

\section{Bibliography}

1. "Diagnostic and statistical manual of mental disorders". $5^{\text {th }}$ edition. Washington, DC [u.a.]: American Psychiatric Publication (2013).

2. Lewis DA and Lieberman JA. "Catching up on schizophrenia: Natural history and neurobiology". Neuron 28.2 (2000): 325334.

3. Bonelli RM and Cummings JL. "Frontal-subcortical circuitry and behavior". Dialogues in Clinical Neuroscience 9.2 (2007): 141-151.

4. Selemon LD and Zecevic N. "Schizophrenia: A tale of two critical periods for prefrontal cortical development". Translational Psychiatry 5.8 (2015): e623.
5. Selemon LD and Goldman-Rakic PS. "The reduced neuropil hypothesis: A circuit based model of schizophrenia". Biological Psychiatry 45.1 (1999): 17-25.

6. Schubert D., et al. "Molecular underpinnings of prefrontal cortex development in rodents provide insights into the etiology of neurodevelopmental disorders". Molecular Psychiatry 20.7 (2015): 795-809.

7. Hoftman GD., et al. "Layer 3 excitatory and inhibitory circuitry in the prefrontal cortex: Developmental trajectories and alterations in schizophrenia". Biological Psychiatry 81.10 (2016): 862-873.

8. McEwen B and Morrison J. "The brain on stress: Vulnerability and plasticity of the prefrontal cortex over the life course". Neuron 79.1 (2013): 16-29.

9. Datta D and Arnsten AFT. "Unique molecular regulation of higher-order prefrontal cortical circuits: Insights into the neurobiology of schizophrenia". ACS Chemical Neuroscience 9.9 (2018): 2127-2145.

10. Perlstein WM., et al. "Relation of prefrontal cortex dysfunction to working memory and symptoms in schizophrenia". American Journal of Psychiatry 158.7 (2001): 1105-1113.

11. Glantz LA and Lewis DA. "Decreased dendritic spine density on prefrontal cortical pyramidal neurons in schizophrenia". Archives of General Psychiatry 57.1 (2000): 65-73.

12. Van Os J., et al. "The environment and schizophrenia". Nature 468.7321 (2010): 203-212.

13. Van Os J., et al. "Gene-environment interactions in schizophrenia: Review of epidemiological findings and future directions". Schizophrenia Bulletin 34.6 (2008): 1066-1082.

14. Hoffmann M. "The human frontal lobes and frontal network systems: An evolutionary, clinical, and treatment perspective". ISRN Neurology (2013): 892459-892434.

15. Miller E and Wallis J. Executive function and higher-order cognition: Definition and neural substrates (2009).

16. Arnsten A and LI B. "Neurobiology of executive functions: Catecholamine influences on prefrontal cortical functions". Biological Psychiatry 57.11 (2004): 1377-1384.

17. Funahashi S and Andreau JM. "Prefrontal cortex and neural mechanisms of executive function". Journal of Physiology - Paris 107.6 (2013): 471-482. 
18. Xing B., et al. "Norepinephrine versus dopamine and their interaction in modulating synaptic function in the prefrontal cortex". Brain Research 1641 (2016): 217-233.

19. Petanjek Z., et al. "The protracted maturation of associative layer IIIC pyramidal neurons in the human prefrontal cortex during childhood: A major role in cognitive development and selective alteration in autism". Frontiers in psychiatry 10 (2019): 122.

20. Birnbaum SG., et al. "Protein kinase $\mathrm{C}$ overactivity impairs prefrontal cortical regulation of working memory". Science 306.5697 (2004): 882-884.

21. Wang M., et al. " $\alpha 2 A$-adrenoceptors strengthen working memory networks by inhibiting CAMP-HCN channel signaling in prefrontal cortex". Cell 129.2 (2007): 397-410.

22. Arnsten AFT. "Stress signalling pathways that impair prefrontal cortex structure and function". Nature Reviews Neuroscience 10.6 (2009): 410-422.

23. Bitanihirwe BKY., et al. "Glutamatergic deficits and parvalbumin-containing inhibitory neurons in the prefrontal cortex in schizophrenia". BMC Psychiatry 9.1 (2009): 71.

24. Goldman-Rakic PS. "Cellular basis of working memory". Neuron 14.3 (1995): 477-485.

25. Ferguson BR and Gao W. "PV interneurons: Critical regulators of E/I balance for prefrontal cortex-dependent behavior and psychiatric disorders". Frontiers in Neural Circuits 12 (2018): 37.

26. Arnsten AFT., et al. "Dynamic network connectivity: A new form of neuroplasticity". Trends in Cognitive Sciences 14.8 (2010): 365-375.

27. Arnsten AFT. "Stress weakens prefrontal networks: Molecular insults to higher cognition". Nature Neuroscience 18.10 (2015): 1376-1385.

28. Gamo NJ., et al. "Stress impairs prefrontal cortical function via D1 dopamine receptor interactions with hyperpolarizationactivated cyclic nucleotide-gated channels". Biological Psychiatry 78.12 (2015): 860-870.

29. Jin LE., et al. "mGluR2/3 mechanisms in primate dorsolateral prefrontal cortex: Evidence for both presynaptic and postsynaptic actions". Molecular Psychiatry 22.11 (2017): 1615-1625.
30. Hains AB., et al. "Chronic stimulation of alpha-2A-adrenoceptors with guanfacine protects rodent prefrontal cortex dendritic spines and cognition from the effects of chronic stress". Neurobiology of Stress 2 (2015): 1-9.

\section{Assets from publication with us}

- Prompt Acknowledgement after receiving the article

- Thorough Double blinded peer review

- Rapid Publication

- Issue of Publication Certificate

- High visibility of your Published work

Website: www.actascientific.com/

Submit Article: www.actascientific.com/submission.php Email us: editor@actascientific.com

Contact us: +919182824667 